Heterogeneous nucleation in hypermonotectic aluminum alloys

This article has been downloaded from IOPscience. Please scroll down to see the full text article.

2012 IOP Conf. Ser.: Mater. Sci. Eng. 27012005

(http://iopscience.iop.org/1757-899X/27/1/012005)

View the table of contents for this issue, or go to the journal homepage for more

Download details:

IP Address: 129.247.247.240

The article was downloaded on 12/01/2012 at 14:52

Please note that terms and conditions apply. 


\title{
Heterogeneous nucleation in hypermonotectic aluminum alloys
}

\author{
M Köhler ${ }^{1}$, L Ratke ${ }^{1}$, I Kaban ${ }^{2}$ and W Hoyer ${ }^{3}$ \\ ${ }^{1}$ Institut für Materialphysik im Weltraum, Deutsches Zentrum für Luft- und Raumfahrt \\ (DLR), D-51170 Köln, Germany \\ ${ }^{2}$ IFW Dresden, Institute for Complex Materials, P.O.B. 270116, D-01171 Dresden, Germany \\ ${ }^{3}$ Chemnitz University of Technology, Institute of Physics, D-09107 Chemnitz, Germany \\ E-mail: markus.koehler@dlr.de
}

\begin{abstract}
Simple casting experiments were set up to solve the question, if heterogeneous nucleation of the liquid-liquid decomposition in monotectic systems is possible. Al-Pb alloys with different inoculants were solidified, and the resulting microstructure was analysed by SEM and X-ray microtomography. Pronounced changes in the distribution of the lead precipitations indicate that it is possible to trigger the nucleation.
\end{abstract}

\section{Introduction}

All monotectic alloys are characterised by a miscibility gap in the liquid state. Although monotectic alloys have been under investigation since a few decades, the essential processes forming the microstructure in these alloys while passing the miscibility gap are not understood. Especially the mechanism of nucleation of liquid-liquid decomposition is currently unknown. Previous experiments and theories [1,2] showed large undercoolings for the decomposition and come to the conclusion, that the process is homogeneous. Recent experiments [3] on multicomponent monotectic aluminum alloys with grain refiners showed distinct changes on the size of precipitations of the minority $L_{2}$ phase.

To further analyse the nucleation process during cooling through the miscibility gap we set up experiments with hypermonotectic $\mathrm{Al}-\mathrm{Pb}$ alloys with grain refiners. The phase diagram of $\mathrm{Al}-\mathrm{Pb}$ is shown in figure 1 . The system exhibits a large miscibility gap spanning over almost the whole concentration range and with temperature boundaries reaching from $1422^{\circ} \mathrm{C}$ (critical point) to $659^{\circ} \mathrm{C}$ (monotectic reaction point).

The classical grain refiner for Al-alloys is $\mathrm{TiBAl} 5 / 1$, which consists mainly of $\mathrm{Al}_{3} \mathrm{Ti}$ (from excess titanium) and $\mathrm{TiB}_{2}$ as active components. Besides $\mathrm{TiBAl}$ the effect of pure $\mathrm{Ti}$ or $\mathrm{TiB}_{2}$ additions was also studied. Inoculation should have an effect on the undercooling needed to form the liquid precipitates after entering the miscibility gap, and therefore have an impact on the microstructure. The particle distribution after solidification of monotectic alloys is different from the point of nucleation because the precipitates are mobile and are susceptible to convection and coagulation. 


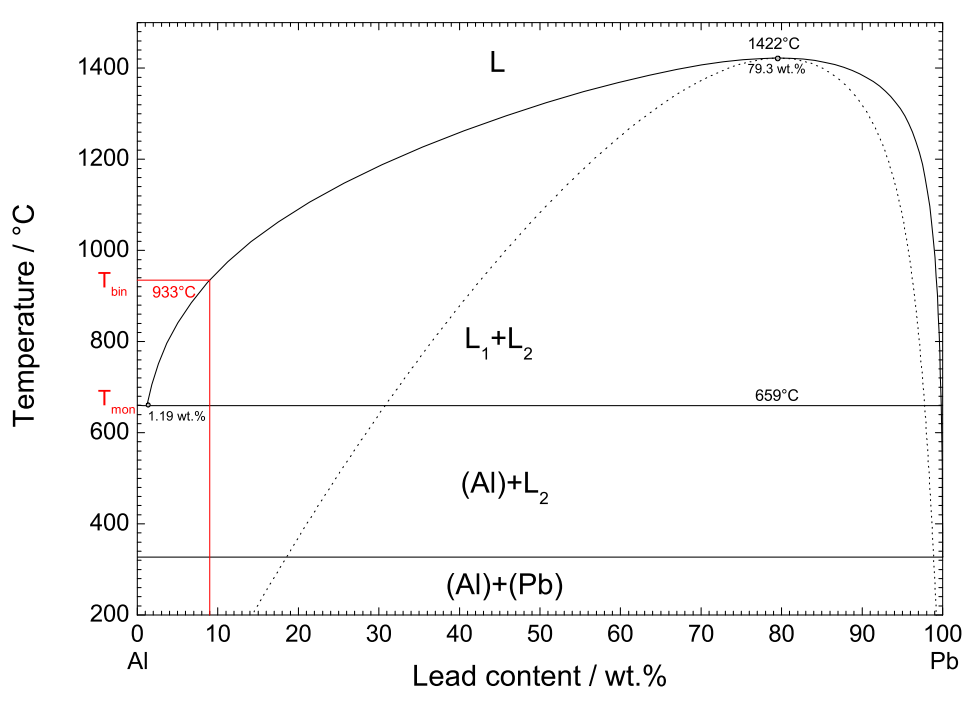

Figure 1. Phase diagram for the examined system $\mathrm{Al}-\mathrm{Pb}$ after $\mathrm{Yu}[4]$ with calculated spinodal line (dashed line). The composition used in this work is marked.

\section{Experimental work}

Aluminum with $9 \mathrm{wt} . \%$ lead was chosen as the base alloy, which is far outside the region of spinodal decomposition. To this $16.7 \mathrm{wt} . \%$ TiBAl $5 / 1$ grain refiner was added, so that the resulting sample contains a total of $1 \mathrm{wt} . \%$ of the active components $\mathrm{Ti}$ and $\mathrm{B}$. Al-10Ti master alloys and $\mathrm{TiB}_{2}$ were added for comparison in separate experiments to study their influence alone. The concentrations were chosen to be comparable to them in the TiBAl samples, and also the double of that concentration was evaluated. A reference sample without inoculants was cast to have a direct comparison.

Samples were produced by melting $\mathrm{Al}$ and $\mathrm{Pb}$ (both purity $\geq 99.99 \%$ ) in a graphite crucible. The melt was held for one hour at a temperature of $1050^{\circ} \mathrm{C}$, while argon was blown into the crucible to stir and degas the melt, and to create an Ar-atmosphere inside. The gas inlet was stopped 5 minutes prior to casting. To trigger nucleation, inoculants, such as TiBAl 5/1 grain refiner, Al-10Ti master alloy (both from LSM) and $\mathrm{TiB}_{2}$ powder, which was first mixed with lead in a ball miller and then compressed, were also added 5 minutes before casting to prevent fading of the inoculant. The casting was done into a $12 \mathrm{~mm}$ diameter bore of a cylindrical steel mould, which had a height of $60 \mathrm{~mm}$ and an outer diameter of $60 \mathrm{~mm}$. To measure the cooling rate, a very thin, fast reacting thermocouple could be placed into center of the bore, and the temperature was recorded on a PC. From several experiments we determined a mean cooling rate of roughly $180 \pm 30 \mathrm{~K} / \mathrm{s}$ for the passage through the miscibility gap.

The solidified sample rod was cut into $5 \mathrm{~mm}$ thick discs. The surface of the disc at a distance of $30 \mathrm{~mm}$ from the bottom was polished and used for microstructure analysis in a SEM. From the center of the disc below this one a small rodlike piece of about $1.5 \mathrm{~mm}$ diameter was extracted and studied by X-ray microtomography. 


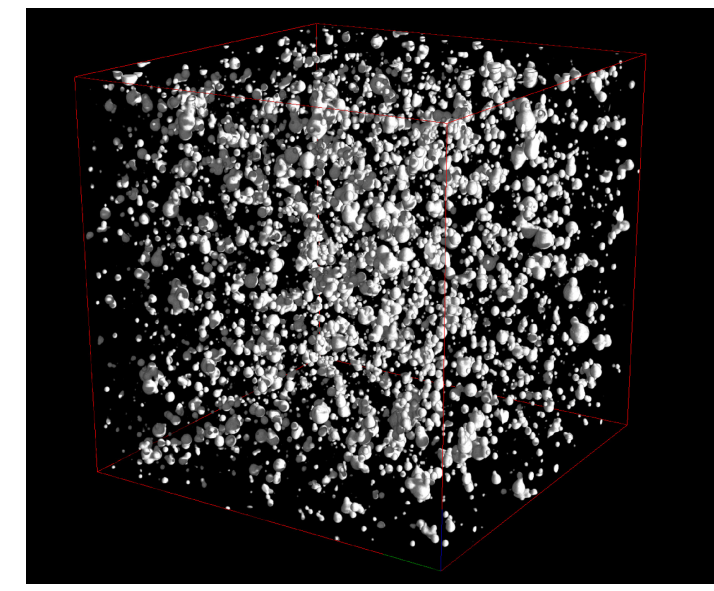

a) reference sample without inoculants

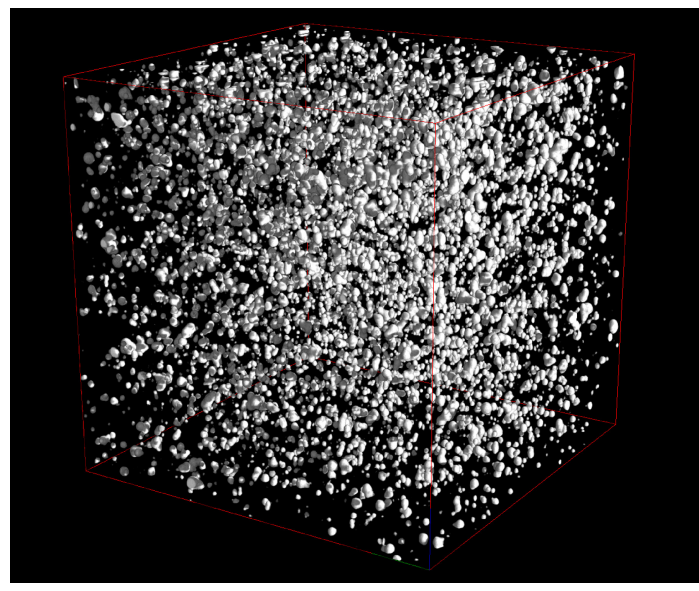

c) $0.53 \% \mathrm{TiB}_{2}$

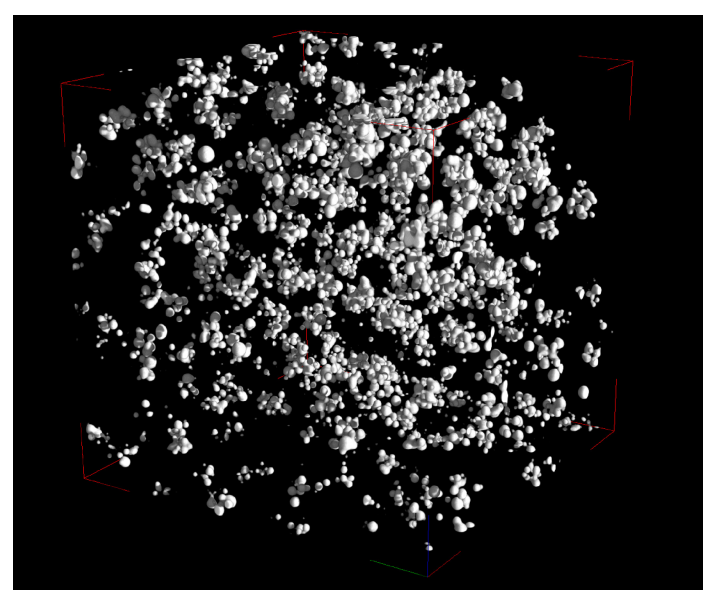

e) $0.47 \% \mathrm{Ti}$

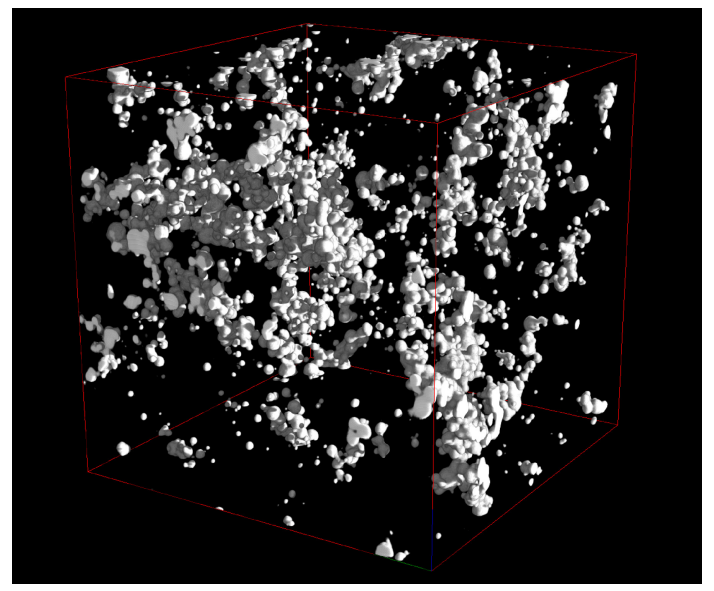

b) $\mathrm{TiBAl} 5 / 1$

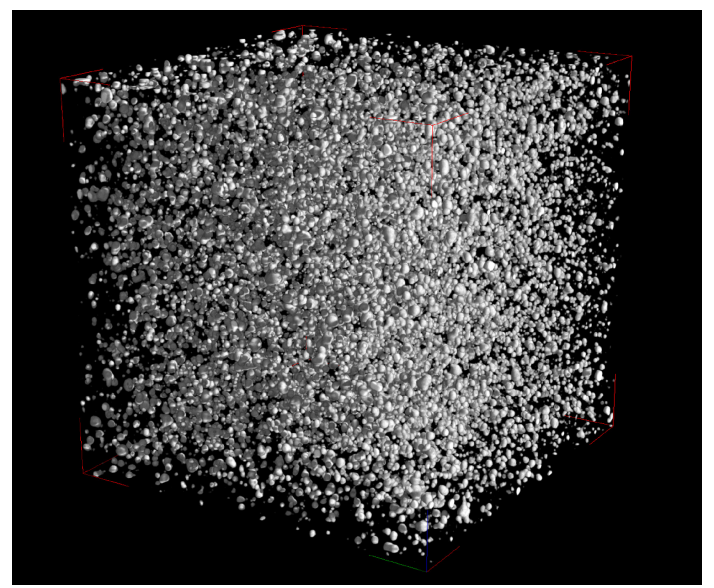

d) $1.00 \% \mathrm{TiB}_{2}$

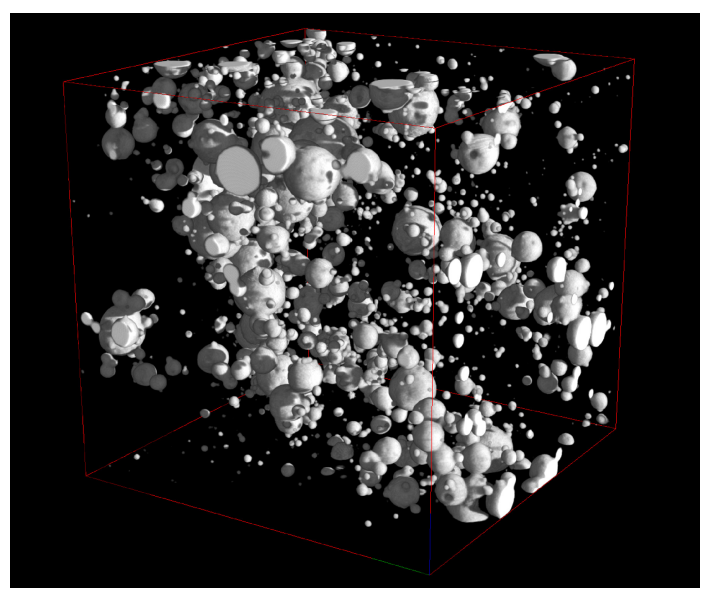

f) $0.94 \% \mathrm{Ti}$

Figure 2. Microtomography of the samples, only the lead particles are displayed. Box size $490 \mu \mathrm{m}$ 


\section{Results}

Figure 2 shows the resulting microstructures as acquired by tomography. On a first look most diverging are the microstructures of the $\mathrm{Ti}$ and TiBAl samples. While the reference sample and the ones with $\mathrm{TiB}_{2}$ show densely packed lead droplets, this is not the case for the samples containing Ti alone. For them a strong clustering can be seen, and the sample with almost one percent of free titanium shows largely oversized particles. But also samples containing $\mathrm{TiB}_{2}$ are different from the reference sample. The particles seem to have a denser packing. Analysing the particle density of the microtomograms shows indeed an increase with $\mathrm{TiB}_{2}$, roughly by a factor of two for the largest addition.

A closer look on the cross section is given by the SEM images shown in figure 3. In addition to the observations of the tomograms, a slight reductions of the maximum droplet diameter by use of $\mathrm{TiB}_{2}$ can be seen. The sample refined with TiBAl shows highly deformed droplets. Another observation for the samples containing excess titanium is that surrounding of the lead droplets is enriched by another type of particles. EDX analysis verifies that those particles are $\mathrm{Al}_{3} \mathrm{Ti}$, formed out of excess Ti during cooling through the miscibility gap.

To obtain the particle size distribution, we applied image analysis on the SEM images. The distribution is plotted in figure 4. The distinct effects of the different inoculants are clearly visible. Additions of $\mathrm{TiB}_{2}$ lead to an increase of the particle number and decrease the width of the distribution. For additions of titanium the distribution is broadened, while the overall particle number is decreased.

For TiBAl the distribution is also shifted to lower diameters and narrower than the reference alloy, but the particle number is less than it is without inoculants.

\section{Discussion}

$\mathrm{TiB}_{2}$ as inoculant seems to have an impressive effect on the lead precipitation. Gröbner et al. [5] have shown, that titaniumdiboride is stable in Al-melts for the temperatures we used in our experiments. When the one-phase liquid passes the binodal, liquid-liquid decomposition can be triggered by the available crystallites, here $\mathrm{TiB}_{2}$. The nucleation potency can be estimated from the wetting angle of $\mathrm{L}_{2}$ in the presence of $\mathrm{L}_{1}$ on polycrystalline $\mathrm{TiB}_{2}$, which has been measured in the $\mathrm{Al}-\mathrm{Pb}$ system on $\mathrm{TiB}_{2}$ by Kaban et al.[6] to be $\approx 21^{\circ}$. With

$$
f(\theta)=\frac{2-3 \cos \theta+\cos ^{3} \theta}{4}
$$

for the catalytic efficiency, we obtain a factor of $0.3 \%$, which means that the barrier for nucleation of $\mathrm{L}_{2}$ in the supercooled single phase Al-Pb liquid is reduced by a factor of 300 . Since inoculation leads to a higher number of droplets, their growth is reduced because of the reduced amount of lead available to each droplet. Smaller particles in general are less susceptible to Marangoni or Stokes motion and coagulation thereby. This might explain the refined distribution of lead droplets on the usage of $\mathrm{TiB}_{2}$.

In the case of Ti as inoculant, we have to take several facts into account. As mentioned above the $\mathrm{Al}_{3} \mathrm{Ti}$ particles are mostly enriched at the perimeter of the lead droplets meaning that $\mathrm{Al}_{3} \mathrm{Ti}$ precipitates are obviously wetted by lead with a wetting angle less than $180^{\circ}$. The catalytic factor for $\theta<180^{\circ}$ is also smaller than one, which reduces the energy barrier for the nucleation process.

The phase diagram of pure $\mathrm{Al}-\mathrm{Ti}$ (figure 5) shows, that $\mathrm{Al}_{3} \mathrm{Ti}$ will be formed upon cooling of an $\mathrm{Al}-\mathrm{Ti}$ melt below the liquidus line. $\mathrm{Al}_{3} \mathrm{Ti}$ precipitates as a pro-peritectic phase. The temperature, at which that happens, is strongly dependent on the concentration of titanium. For the lower concentration of titanium $(0.47 \%)$ the liquidus line is crossed at $790^{\circ} \mathrm{C}$, while for the higher concentration the liquidus line is already passed at $890^{\circ} \mathrm{C}$. The binodal temperature 
The 3rd International Conference on Advances in Solidification Processes IOP Publishing IOP Conf. Series: Materials Science and Engineering 27 (2011) 012005

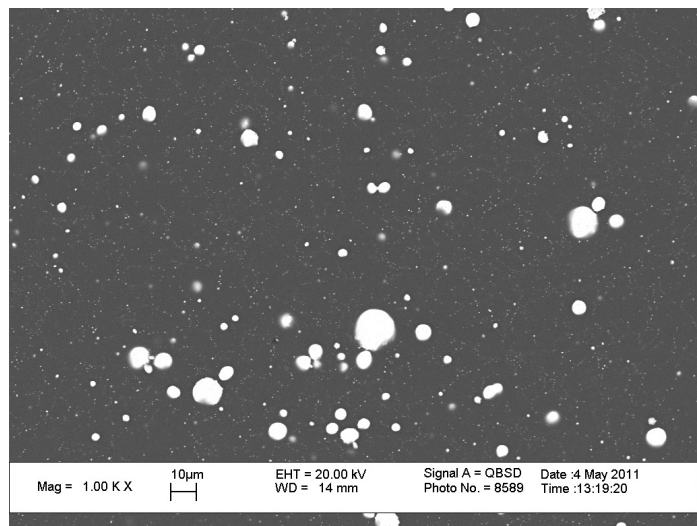

a) reference sample without inoculants

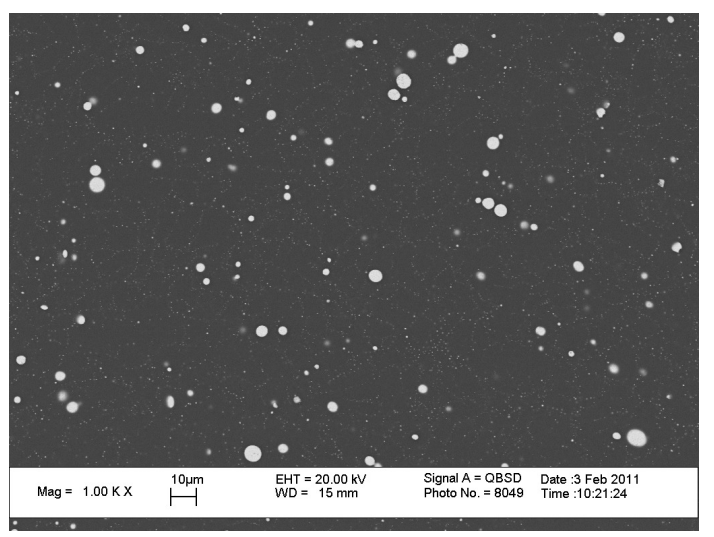

c) $0.53 \% \mathrm{TiB}_{2}$

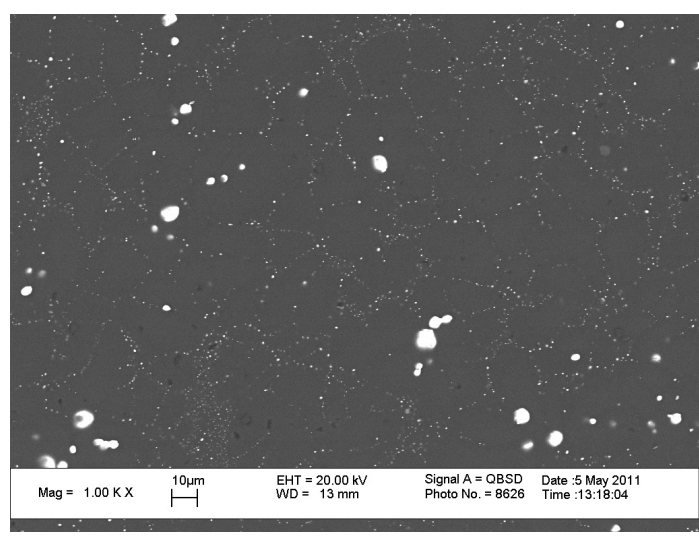

e) $0.47 \% \mathrm{Ti}$

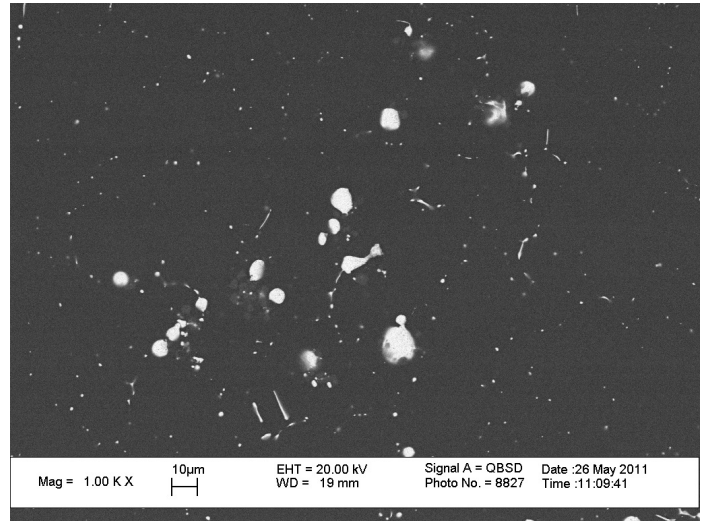

b) $\mathrm{TiBAl} 5 / 1$

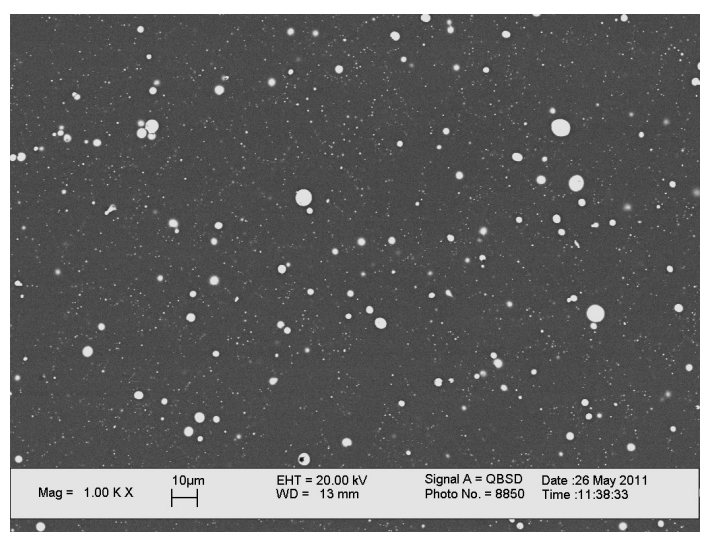

d) $1.00 \% \mathrm{TiB}_{2}$

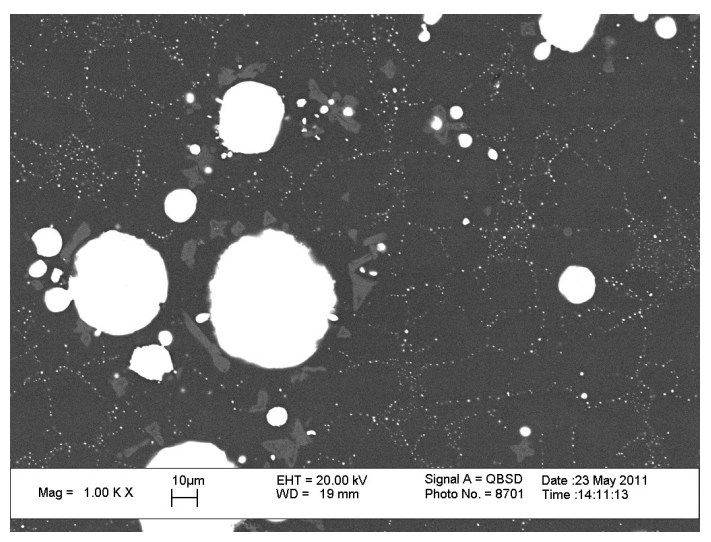

f) $0.94 \% \mathrm{Ti}$

Figure 3. SEM images of the samples. Lead particles are white, the Al matrix is dark grey and $\mathrm{Al}_{3} \mathrm{Ti}$ particles are light grey 


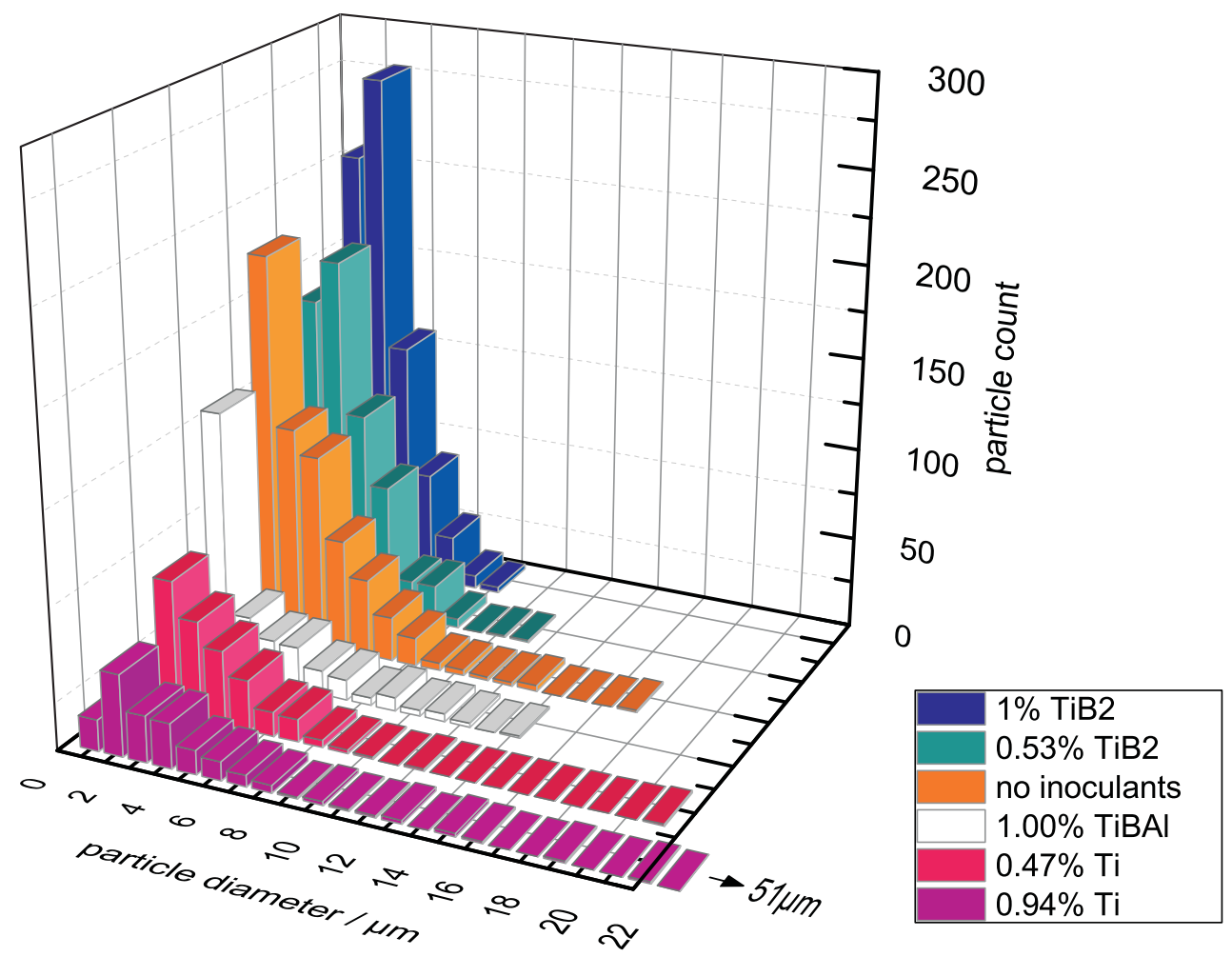

Figure 4. Size distribution of the lead precipitates acquired from SEM data.

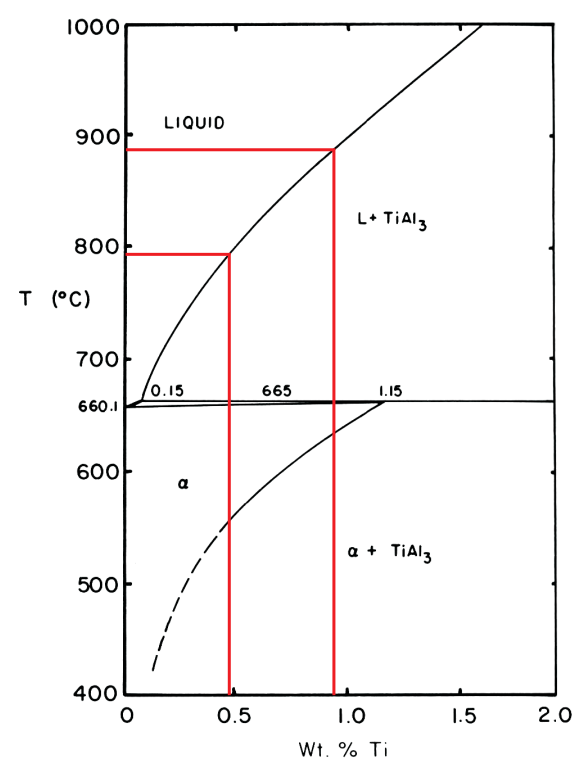

Figure 5. Aluminum rich corner of the Al-Ti phase diagram by Sigworth [7]. The used concentrations of titanium are marked. 
of $\mathrm{Al}-9 \mathrm{~Pb}$ is at $933^{\circ} \mathrm{C}$. We assume, that those points in the ternary phase diagram are only slightly altered compared to the binary alloys.

For $\mathrm{Al}_{3} \mathrm{Ti}$ to have an effect on the nucleation, it has to reach an undercooling for the liquid-liquid decomposition of about $140 \mathrm{~K}$, or still $40 \mathrm{~K}$ for the higher Ti content. Assuming heterogeneous nucleation is possible, these undercoolings are not realistic. It is more probable, that first the lead precipitates will form, then the $\mathrm{Al}_{3} \mathrm{Ti}$ is nucleated on these precipitates. More experiments with more different titanium contents are neccessary to evaluate this effect further.

The impact of TiBAl on the microstructure can be interpreted as a superposition of the two previous effects. Nucleation is stimulated by the available $\mathrm{TiB}_{2}$. In later stages those lead droplets are covered by $\mathrm{Al}_{3} \mathrm{Ti}$ and therefore cannot act as nucleation centers for lead anymore and such a coverage reduces drastically an Marangoni motion of droplets and coagulation thereby [8]. So the overall droplet density is less compared to samples containing $\mathrm{TiB}_{2}$.

The effect TiBAl and $\mathrm{Al}_{3} \mathrm{Ti}$ have as a grain refiner for $\alpha$-aluminum has to be considered separately. The $\mathrm{Al}_{3} \mathrm{Ti}$ enhances nucleation of $\alpha$-Al grains [9] in samples containing solute titanium as the pure $\mathrm{Ti}$ and $\mathrm{TiBAl}$ inoculated samples. The role of this effect has not been disclosed in detail, but there might be an influence on the final monotectic reaction. In the monotectic reaction the Al-rich liquid decomposes at the monotectic temperature into solid $\alpha$-aluminum and $\mathrm{L}_{2}$ droplets. If the primary $\alpha$-aluminum and thus the monotectic reaction is initiated by $\mathrm{Al}_{3} \mathrm{Ti}$, the monotectic droplets might be shifted by the growing primary phase dendrites, are enriched at the boundaries of the equiaxed dendritic grains and can coagulate with the droplets stemming from passing the miscibility gap. Therefore, an increase in droplet size could be expected as observed.

\section{Conclusion}

Inoculants have an effect on the liquid-liquid decomposition in $\mathrm{Al}-\mathrm{Pb}$ alloys during cooling through the miscibility gap. Especially effective is $\mathrm{TiB}_{2}$; less effective is $\mathrm{Al}_{3} \mathrm{Ti}$ which seems to decorate the $\mathrm{Pb}$ droplets, possibly formed via a homogeneous nucleation route. The microstructure is characterised by a clustering of droplets. Conventional grain refiners like TiBAl are more complex in their cause-effect-chain. Generally we conclude that the liquidliquid decomposition can be initiated by heterogeneous nucleation process. If the homogeneous nucleation route also is possible remains an open question.

\section{References}

[1] Uebber N and Ratke L 1991 Scripta Metallurgica et Materialia 25 1133-7

[2] Grnsy L and Ratke L 1993 Scripta Metallurgica et Materialia 28 1329-34

[3] Ratke L, Brck S, Mathiesen R, Ludwig A, Gruber-Pretzler M, Tonn B, Gzovskyy K, Grnsy L, Tegze G, gren J, Hglund L, Arnberg L, Gust E, Anger G, Lauer M, Garen R and Reifenhuser B 2007 Transactions of the Indian Institute of Metals 60 103-11

[4] Yu S K, Sommer F and Predel B 1996 Zeitschrift fr Metallkunde 87 574-80

[5] Grbner J, Mirkovi D and Schmid-Fetzer R 2005 Materials Science and Engineering A 395 10-21

[6] Kaban I, Khler M, Hoyer W and Ratke L 2010 High Temperatures - High Pressures $39347-55$

[7] Sigworth G 1984 Metallurgical and Materials Transactions A 15(2) 277-82

[8] Subramanian R and Balasubramaniam R 2001 The motion of bubbles and drops in reduced gravity (Cambridge University Press, New York)

[9] Quested T E 2004 Materials Science and Technology 20 1357-1369 